\title{
Magnetic resonance imaging versus echocardiography to ascertain the regression of left ventricular hypertrophy after bioprosthetic aortic valve replacement: Results of the REST study
}

\author{
Ingo Breitenbach, MD, ${ }^{\mathrm{a}}$ Wolfgang Harringer, $\mathrm{MD},{ }^{\mathrm{a}}$ Steven Tsui, $\mathrm{MD},{ }^{\mathrm{b}}$ Mario Jorge Amorim, $\mathrm{MD},{ }^{\mathrm{c}}$ \\ Marie-Christine Herregods, MD, PhD, ${ }^{\mathrm{d}}$ Jan Bogaert, MD, ${ }^{\mathrm{e}}$ Juan Jose Goiti, MD, ${ }^{\mathrm{f}}$ and Gino Gerosa, $\mathrm{MD}^{\mathrm{g}}$
}

Objectives: To compare the decrease in left ventricular mass index (LVMI) by magnetic resonance imaging (MRI) versus transthoracic echocardiography (TTE) after aortic valve replacement (AVR) for severe aortic stenosis with Epic and Epic Supra stented porcine bioprostheses (St Jude Medical, Inc, St Paul, Minn).

\begin{abstract}
Methods: This prospective multicenter study enrolled 149 patients who underwent AVR between January 2006 and February 2008. TTE and cardiac MRI measurements of LVMI were made at baseline and at 6 months of follow-up and were compared. Changes in mean pressure gradients were examined using TTE.
\end{abstract}

Results: TTE measurements of LVMI were $48 \%$ to $63 \%$ higher than the MRI measurements. A decrease in LVMI from $137 \pm 32$ to $95 \pm 16 \mathrm{~g} / \mathrm{m}^{2}$ with the Epic and from $139 \pm 29$ to $104 \pm 28 \mathrm{~g} / \mathrm{m}^{2}$ with the Epic Supra valves $(P<.0001$ for both comparisons) was measured by TTE. Cardiac MRI revealed decreases in LVMI from $84 \pm 20$ to $64 \pm 12 \mathrm{~g} / \mathrm{m}^{2}$ and from $86 \pm 27$ to $64 \pm 17 \mathrm{~g} / \mathrm{m}^{2}$ with the Epic and Epic Supra valves, respectively $(P<.0001$ for both comparisons). TTE revealed a significant regression of mean pressure gradients from $51.6 \pm$ 15.3 to $15.5 \pm 5.2 \mathrm{~mm} \mathrm{Hg}$ with the Epic and from $46.7 \pm 19.4$ to $17.9 \pm 12.8 \mathrm{~mm} \mathrm{Hg}$ with the Epic supra $(P<.0001$ for both comparisons).

Conclusions: A significant decrease in LVMI was measured after AVR with all sizes of both bioprosthetic models. Because of the overestimation of the decrease in LVMI by the Devereux formula, as well as the higher accuracy and reproducibility of cardiac MRI measurements, the latter should be preferred to TTE. An ultimate validation of this thesis could only be done comparing each of these modalities with pathologic examination. (J Thorac Cardiovasc Surg 2012;144:640-5)

Supplemental material is available online.

Aortic valve replacement (AVR) remains the main treatment of severe aortic stenosis (AS). ${ }^{1}$ Several studies have confirmed the benefit it confers on long-term survival, symptomatic relief, and quality of life, ${ }^{2,3}$ a benefit

From the Department of Cardiac, ${ }^{\text {a }}$ Thoracic and Vascular Surgery, Klinikum Braunschweig, Braunschweig, Germany; Department of Cardiothoracic Surgery, ${ }^{\mathrm{b}}$ Papworth Hospital, Papworth Everard, Cambridge, United Kingdom; Department of Cardiothoracic Surgery, ${ }^{\mathrm{c}}$ Hospital de São João, Porto, Portugal; Department of Cardiovascular Diseases, ${ }^{d}$ Katholieke Universiteit Leuven, Leuven, Belgium; Department of Radiology, ${ }^{\mathrm{e}}$ University of Leuven, Gasthuisberg Universital Hospital, Leuven, Belgium; Department of Cardiovascular Surgery, ${ }^{\mathrm{f}}$ Policlinica Gipuzkoa, San Sebastian, Spain; and Division of Cardiac Surgery, ${ }^{\mathrm{g}}$ Department of Cardiac, Thoracic and Vascular Sciences, Padua University, Padua, Italy.

This study was sponsored by St Jude Medical EMEAC. ClinicalTrials.gov Identifier: NCT00256165.

Disclosures: Authors have nothing to disclose with regard to commercial support. Received for publication Aug 3, 2011; revisions received Oct 8, 2011; accepted for publication Nov 8, 2011; available ahead of print Dec 12, 2011.

Address for reprints: Ingo Breitenbach, MD, Department of Cardiac, Thoracic and Vascular Surgery, Klinikum Braunschweig, Salzdahlumerstr. 90, 38126 Braunschweig, Germany (E-mail: ibreitenbach@me.com).

$0022-5223 / \$ 36.00$

Copyright (c) 2012 by The American Association for Thoracic Surgery doi:10.1016/j.jtcvs.2011.11.017 attributable to a decrease in left ventricular (LV) pressure overload, allowing the decrease in LV mass and regression of hypertrophy.

LV mass is an independent predictor of cardiovascular morbidity and mortality. ${ }^{4}$ It is crucial in patients who have severe, symptomatic AS to lower the LV afterload and normalize the LV mass, a process that takes place mainly within the first 3 postoperative months. However, significant LV hypertrophy persists in a subset of patients. ${ }^{5}$ Systemic hypertension and a small prosthetic valve are 2 causes of persistent LV hypertrophy after surgical AVR, ${ }^{6,7}$ underscoring the importance of an optimal postoperative treatment plan to (1) achieve the greatest amount of decrease in LV mass and (2) most reliably ascertain its changes.

Most studies of LV mass and its decrease after AVR have used transthoracic echocardiography (TTE), a method that is readily available and that uses geometric assumptions that have been validated in normal hearts. ${ }^{8}$ However, in the presence of asymmetric LV hypertrophy, the LV mass may be overestimated. ${ }^{9,10}$ Moreover TTE is (1) dependent on the acoustic window and (2) subject to interobserver and intraobserver variability. ${ }^{11}$ In contrast, cardiovascular magnetic resonance imaging (MRI) enables the acquisition of high-resolution images in any plane, 


\author{
Abbreviations and Acronyms \\ AS $=$ aortic stenosis \\ AVR $=$ aortic valve replacement \\ $\mathrm{CABG}=$ coronary artery bypass graft \\ $\mathrm{LV}=$ left ventricular \\ LVMI $=$ left ventricular mass index \\ MRI = magnetic resonance imaging \\ REST $=$ Left Ventricular Regression European \\ Study \\ TTE $=$ transthoracic echocardiography
}

unlimited by an acoustic window and without exposure to radiation, producing measurements that are accurate and highly reproducible.

The objective of this prospective multicenter study was to compare the decrease in LV mass measured by MRI versus that measured by TTE, 6 months after AVR, using the Epic and Epic Supra bioprostheses (St Jude Medical, Inc, St Paul, Minn).

\section{STUDY POPULATION AND METHODS}

The Left Ventricular Regression European STudy (REST) was conducted at 8 European medical centers. The data were collected by study coordinators appointed at each participating center, analyzed by an independent core laboratory, and monitored by St Jude Medical, the study sponsor. The protocol was approved by the research ethics committee of each participating center, and all patients granted their written informed consent to participate in the study.

\section{Sample Size Calculation}

The sample size calculation was based on the main end point of the study, that is, change in LV mass index (LVMI) between baseline and 6 months. Baseline is defined as before intervention. According to BechHanssen and associates, ${ }^{12}$ it was assumed that the mean change of LVMI using echocardiography for all biological valves will be $18.35 \%$ with an estimated standard deviation of $17.35 \%$.

For sample size calculations, it was assumed that the mean change of LVMI using MRI will be $25 \%$, with the same standard deviation as the one for mean change of LVMI using echocardiography, that is, $17.35 \%$. Moreover, several values were assumed for the correlation between the change in LVMI using MRI and using echocardiography. Assuming a very weak association of 0.2 , a 2 -sided paired $t$ test will detect a significant difference (5\% significance level and power at least $90 \%$ ) in change of LVMI between echocardiography and MRI when 117 analyzable patients are recruited. If the correlation is 0.4 , then 88 patients should be recruited. If the correlation is higher, the sample size required will decrease.

\section{Study Eligibility}

Patients of legal age in their host country, who underwent a first AVR for severe AS or for mixed aortic valve disease with predominant AS, were considered for study enrollment. In February 2007, an amendment to the protocol was introduced, which allowed the enrollment of patients undergoing AVR with concomitant coronary artery bypass graft (CABG) surgery. Patients were excluded from the study if they were pregnant or nursing, on hemodialysis, or had unstable angina, active endocarditis, acute aortic dissection, New York Heart Association heart failure functional class
IV, or prominent LV wall motion abnormality, chronic or persistent atrial fibrillation, or claustrophobia. Further exclusion criteria were the presence of a permanent pacemaker or implantable cardioverter defibrillator, aneurysmal clip, carotid artery stent, neurostimulator, cochlear or other ear implant, temporary or permanently implanted drug infusion device, bone growth or fusion stimulator, and ocular foreign body.

\section{Study Design}

This was an international, multicenter, prospective, observational study. Demographic and clinical characteristics, health history, concomitant medications, and baseline laboratory values were recorded in all patients. The patients were scheduled to return for ambulatory follow-up visits at 6-month (time frame from 180 to 270 days) intervals after their discharge from the hospital. TTE and MRI were performed at baseline and at 6 months of follow-up.

\section{Implanted Valve Models}

Epic and Epic Supra are stented porcine bioprostheses. The choice of Epic versus Epic Supra valve implanted in this study was left to the surgeon's discretion.

\section{TTE}

All TTEs were performed by expert sonographers at site, trained on study echocardiography protocol, and analyzed at an independent core laboratory (U.Z. Gasthuisberg, Department of Cardiology, Leuven, Belgium) by observers highly trained in qualitative, quantitative, and Doppler ultrasonography. Two-dimensional, M-mode, and Doppler images were obtained in standard apical, subcostal, suprasternal, and parasternal views. Measurements were performed according to the guidelines issued by the American Society of Echocardiography. ${ }^{13}$ LV mass was calculated using the Devereux formula.

\section{Cardiac MRI}

MRI was performed during continuous electrocardiographic monitoring using 5 different scans. The new, balanced, steady-state free precession cine MRI sequences were used for the measurements of LV volumes, mass, and function in scans 3, 4, and 5. For the cine MRIs in the short axis, an 8-mm thick continuous slice was used with a repetition time of $35 \mathrm{~ms}$ or less. LV end-diastolic volume, LV end-systolic volume, stroke volume, LV mass, and their associated indices, and LV ejection fraction were calculated by an independent MRI core laboratory (U.Z. Gasthuisberg, Department of Radiology, Leuven, Belgium) using a custom-made software (CardioViewer; Catholic University Leuven, Leuven, Belgium). ${ }^{14}$ The endocardial trabeculations and papillary muscles were included in the LV wall measurements.

\section{Statistical Analysis}

Continuous variables are presented as means \pm standard deviation and were compared with the Student $t$ test or Wilcoxon nonparametric test, as appropriate. Categorical data are presented as counts and percentages. The relationship between measurements of LV mass and LVMI by MRI versus echocardiography was examined using the Spearman rank correlation coefficients and associated $P$ values. All statistical analyses were performed with SAS software, version 9.1 (SAS Institute Inc, Cary, NC).

\section{RESULTS}

\section{Study Population}

A total of 149 patients were enrolled in the REST study between January 2006 and June 2008. Their mean age was $73.3 \pm 8.6$ years and $57 \%$ were women. The main baseline characteristics of the study population are shown in Table 1. 
TABLE 1. Baseline characteristics of the 149 study patients

\begin{tabular}{lc}
\hline Age, $\mathrm{y}$ & $73.3 \pm 8.6$ \\
Women & $85(57)$ \\
Height, $\mathrm{cm}$ & $164.2 \pm 9.7$ \\
Body & \\
$\quad$ Weight, $\mathrm{kg}$ & $75.1 \pm 14.1$ \\
$\quad$ Mass index, $\mathrm{kg} / \mathrm{m}^{2}$ & $27.9 \pm 4.9$ \\
$\quad$ Surface area, ${ }^{2}$ & $1.8 \pm 0.2$ \\
Log EuroSCORE & $6.6 \pm 4.0$ \\
New York Heart Association functional class & \\
$\quad$ I & $21(14 \%)$ \\
II & $86(58 \%)$ \\
$\quad$ III & $42(28 \%)$ \\
\hline Values are means \pm standard deviation or numbers $(\%)$ of observations.
\end{tabular}

\section{Surgical and Intraoperative Observations}

Fifty-four (36\%) patients had pure AS and $95(64 \%)$ had aortic insufficiency and predominant stenosis (Table 2). The peak and mean pressure gradients at baseline were $81 \pm 28$ and $49 \pm 18 \mathrm{~mm} \mathrm{Hg}$, respectively, and mean effective orifice area was $0.80 \pm 0.34 \mathrm{~cm}^{2}$.

Of the 149 patients, 109 underwent isolated AVR, 26 underwent concomitant CABG, and 22 underwent other additional procedures (Table 2). The sizes of the 77 Epic and 72 Epic Supra bioprostheses implanted in this study are detailed in Table 2. The mean duration of cardiopulmonary bypass was $96 \pm 34$ minutes and mean duration of crossclamping was $70 \pm 24$ minutes. Further intraoperative observations and measurements are shown in Table 2.

\section{Postoperative Outcomes and Analyzable Data}

The short- and long-term clinical outcomes and ultimate data availability are shown in Table E1.

Short-term outcome. The overall duration of ventilator support ranged from 5 to 53 hours, the mean stay in the intensive care unit ranged from 6 to 163 hours, and the duration of hospitalization ranged from 5 to 76 days. At 30 days after the operation, $3(2.0 \%)$ of the 149 patients had died, including 1 patient on postoperative day 9 of ventricular fibrillation, 1 patient on day 11 of acute cardiac decompensation and pulmonary edema, and 1 patient on day 12 of unwitnessed sudden death. Major intrathoracic hemorrhages requiring reoperations occurred in $4(2.7 \%)$ patients and hemorrhages treated with transfusions of blood products in $3(2.0 \%)$ patients. Atrial fibrillation developed in 12 $(8.1 \%)$ and complete heart block requiring permanent pacing in $8(5.3 \%)$ patients. Other complications included a major hemorrhage on postoperative day 6 from a duodenal ulcer in $1(0.7 \%)$ patient and large pericardial effusions in 3 $(2.0 \%)$ patients, treated with diuretics, open drainage, and pericardiocentesis in 1 patient each.

Long-term outcome. Over a mean follow-up of 7.1 months and cumulative follow-up of 1052 months, 2 additional patients died, of renal failure and liver cirrhosis,
TABLE 2. Intraoperative observations

\begin{tabular}{lc} 
Valve sizes, mm & \\
Epic Supra & \\
19 & $10(7)$ \\
21 & $38(26)$ \\
23 & $24(16)$ \\
Epic & \\
21 & $7(5)$ \\
23 & $39(26)$ \\
25 & $22(15)$ \\
27 & $9(6)$ \\
Aortic valve lesions & \\
Stenosis & $54(36)$ \\
Mixed stenosis and insufficiency & $95(64)$ \\
Calcifications & \\
Annular & $97(65)$ \\
Leaflet & $134(90)$ \\
Leaflet thickening & $149(100)$ \\
Commissural fusion & $47(32)$ \\
Concomitant procedures & \\
None & $109(73)$ \\
Coronary artery bypass graft & $26(17)$ \\
Aortoplasty & $11(7)$ \\
Replacement of ascending aorta & $2(1)$ \\
Other & $9(6)$ \\
Duration, min & \\
Cardiopulmonary bypass & \\
Crossclamp & $96 \pm 34$ \\
\hline
\end{tabular}

Values are means \pm standard deviation or numbers (\%) of observations.

respectively, for an overall mortality of $3.4 \%$. Valve thrombosis treated with cleaning of the valve leaflets on postoperative day 102 and embolic stroke on postoperative day 116 occurred in 1 patient each. Furthermore, 7 patients withdrew from the study, 1 was lost to follow-up, 2 had pacemakers implanted, and 13 patients had incomplete or untimely collections of data (Table E1). At the end of follow-up, complete and paired echocardiographic and MRI data were available in 109 patients (complete MRI data in 111 patients and complete echocardiographic data in 118 patients).

\section{Echocardiographic Observations at Baseline Versus 6 Months of Follow-up}

The changes in echocardiographic measurements associated with each valve model and each valve size between baseline and 6 months of follow-up are summarized in Tables 3 and 4. LV mass and LVMI both decreased significantly with each model and each valve size (Table 3 and Table 4). Likewise, LV end-diastolic diameter, LV end-diastolic posterior wall and septal thickness, and LV end-diastolic volume decreased significantly. LV endsystolic volume, however, decreased significantly in the Epic group only. Mean LV ejection fraction was normal at baseline in both valve model groups and increased 
TABLE 3. Echocardiographic and MRI observations at baseline versus 6 months of follow-up

\begin{tabular}{|c|c|c|c|c|c|c|c|}
\hline \multirow[b]{2}{*}{ Measurements } & \multirow[b]{2}{*}{ Valve model } & \multicolumn{3}{|c|}{ Echocardiography } & \multicolumn{3}{|c|}{ MRI } \\
\hline & & Baseline & $6 \mathrm{mo}$ & $P$ & Baseline & $6 \mathrm{mo}$ & $P$ \\
\hline \multicolumn{8}{|l|}{ Left ventricular } \\
\hline \multirow[t]{2}{*}{ Mass, g } & Epic & $258 \pm 65$ & $178 \pm 37$ & $<.0001$ & $160 \pm 41$ & $120 \pm 28$ & $<.0001$ \\
\hline & Epic supra & $243 \pm 51$ & $180 \pm 47$ & $<.0001$ & $151 \pm 47$ & $110 \pm 30$ & $<.0001$ \\
\hline \multirow[t]{2}{*}{ Mass index, $\mathrm{g} / \mathrm{m}^{2}$} & Epic & $137 \pm 32$ & $95 \pm 16$ & $<.0001$ & $84 \pm 20$ & $64 \pm 12$ & $<.0001$ \\
\hline & Epic supra & $139 \pm 29$ & $104 \pm 28$ & $<.0001$ & $86 \pm 27$ & $64 \pm 17$ & $<.0001$ \\
\hline \multicolumn{8}{|l|}{ End-distolic, mm } \\
\hline \multirow[t]{2}{*}{ Diameter } & Epic & $45.9 \pm 5.2$ & $43.0 \pm 4.0$ & $<.0001$ & & & \\
\hline & Epic supra & $44.3 \pm 5.5$ & $40.7 \pm 5.7$ & $<.0001$ & & & \\
\hline \multicolumn{8}{|l|}{ Thickness } \\
\hline \multirow[t]{2}{*}{ Posterior wall } & Epic & $12.9 \pm 1.3$ & $10.9 \pm 1.0$ & $<.0001$ & & & \\
\hline & Epic supra & $12.9 \pm 1.4$ & $11.4 \pm 1.3$ & $<.0001$ & & & \\
\hline \multirow[t]{2}{*}{ Septum } & Epic & $14.8 \pm 2.0$ & $12.3 \pm 1.6$ & $<.0001$ & & & \\
\hline & Epic supra & $14.8 \pm 1.8$ & $13.1 \pm 2.1$ & $<.0001$ & & & \\
\hline \multicolumn{8}{|l|}{ Volume, $\mathrm{cm}^{3}$ (TTE) } \\
\hline \multicolumn{8}{|l|}{ Volume, mL (MRI) } \\
\hline \multirow[t]{2}{*}{ End-ddiastolic } & Epic & $110 \pm 29$ & $98 \pm 17$ & $<.0001$ & $131 \pm 36$ & $117 \pm 23$ & .01 \\
\hline & Epic supra & $104 \pm 36$ & $92 \pm 23$ & .026 & $125 \pm 47$ & $110 \pm 28$ & .002 \\
\hline \multirow[t]{2}{*}{ End-systolic } & Epic & $35 \pm 16$ & $31 \pm 10$ & .0032 & $45 \pm 29$ & $44 \pm 31$ & $<.0001$ \\
\hline & Epic supra & $32 \pm 18$ & $28 \pm 10$ & .066 & $30 \pm 13$ & $29 \pm 20$ & $<.0001$ \\
\hline \multirow[t]{2}{*}{ Ejection fraction, $\%$} & Epic & $68.8 \pm 6.3$ & $68.7 \pm 6.8$ & .19 & $67.7 \pm 12.5$ & $74.4 \pm 8.1$ & $<.0001$ \\
\hline & Epic supra & $68.2 \pm 8.8$ & $70.6 \pm 5.1$ & .002 & $68.9 \pm 14.6$ & $74.9 \pm 10.4$ & $<.0001$ \\
\hline \multicolumn{8}{|l|}{ Transvalvular pressure gradient, $\mathrm{mm} \mathrm{Hg}$} \\
\hline \multirow[t]{2}{*}{ Peak } & Epic & $85.2 \pm 25.2$ & $28.5 \pm 10.7$ & $<.0001$ & & & \\
\hline & Epic supra & $77.3 \pm 30.0$ & $31.9 \pm 18.3$ & $<.0001$ & & & \\
\hline \multirow[t]{2}{*}{ Mean } & Epic & $51.6 \pm 15.3$ & $15.5 \pm 5.2$ & $<.0001$ & & & \\
\hline & Epic supra & $46.7 \pm 19.4$ & $17.9 \pm 12.8$ & $<.0001$ & & & \\
\hline \multirow[t]{2}{*}{ Effective orifice area, $\mathrm{cm}^{3}$} & Epic & $0.8 \pm 0.3$ & $1.4 \pm 0.3$ & $<.0001$ & & & \\
\hline & Epic supra & $0.8 \pm 0.4$ & $1.3 \pm 0.3$ & $<.0001$ & & & \\
\hline \multirow[t]{2}{*}{ Effective orifice area index, $\mathrm{cm}^{3} / \mathrm{m}^{2}$} & Epic & $0.4 \pm 0.1$ & $0.8 \pm 0.2$ & $<.0001$ & & & \\
\hline & Epic supra & $0.5 \pm 0.2$ & $0.7 \pm 0.2$ & $<.0001$ & & & \\
\hline \multirow[t]{2}{*}{ Cardiac output, L/min } & Epic & $5.3 \pm 1.6$ & $4.5 \pm 1.2$ & $<.0001$ & $5.9 \pm 1.4$ & $5.6 \pm 1.3$ & .62 \\
\hline & Epic supra & $5.3 \pm 1.2$ & $4.5 \pm 1.2$ & .0039 & $5.3 \pm 1.4$ & $5.3 \pm 1.2$ & .67 \\
\hline \multirow[t]{2}{*}{ Cardiac index, $\mathrm{L} \cdot \min ^{-1} \cdot \mathrm{m}^{-2}$} & Epic & $2.8 \pm 0.8$ & $2.4 \pm 0.6$ & $<.0001$ & $3.2 \pm 0.8$ & $3.0 \pm 0.7$ & .72 \\
\hline & Epic supra & $3.0 \pm 0.7$ & $2.6 \pm 0.7$ & .0079 & $3.0 \pm 0.8$ & $3.1 \pm 0.6$ & .76 \\
\hline
\end{tabular}

Values are means \pm standard deviation. Paired transthoracic echocardiographic (TTE) observations were available in 58 recipients of Epic and 60 recipients of Epic Supra bioprostheses. Paired magnetic resonance imaging (MRI) observations were available in 53 recipients of Epic and 58 recipients of Epic Supra bioprostheses.

significantly from $68.2 \% \pm 8.8 \%$ to $70.6 \% \pm 5.1 \%$ $(P=.002)$ in the Epic Supra group only (Table 3).

\section{Cardiac MRI Observations at Baseline Versus 6 Months of Follow-up}

At 6 months of follow-up, LV mass and LVMI $(P<.0001)$, LV end-diastolic volume $(P<.0001)$ and $\mathrm{LV}$ end-systolic volume $(P=.003)$ had decreased and LV ejection fraction had increased $(P<.0001)$ significantly with both valve models and each valve size (Tables 3 and 4 ). Cardiac output and cardiac index remained unchanged with both valve models (Table 3 ).

\section{Decrease in LV Mass on TTE Versus Cardiac MRI}

Despite moderate correlations observed at baseline $(r=0.54)$ and at 6 months of follow-up $(r=0.62)$ between the LVMI measured by TTE versus MRI, the measurements made by TTE were systematically and significantly higher $(P<.0001)$ than the measurements made by MRI (Table 3 ).

\section{Effects of Concomitant CABG Surgery}

In February 2007, a protocol amendment was introduced allowing the enrolment in REST of patients undergoing AVR and concomitant CABG who had severe AS and no major LV wall motion abnormality. A comparison of the demographic characteristics of 26 patients who underwent concomitant CABG, versus 123 patients who did not, revealed no significant difference (Table E2). Likewise, no differences were observed between the 2 groups in (1) TTE-measured baseline LV ejection fraction, (2) TTEmeasured LV mass at 6 months and at follow-up, and (3) 
TABLE 4. Regression of LV mass and LVMI by valve type and size

\begin{tabular}{|c|c|c|c|c|c|c|c|}
\hline \multirow[b]{2}{*}{ Measurements } & \multicolumn{7}{|c|}{ Valve type and size in $\mathbf{~ m m}$} \\
\hline & Epic 21 & Epic 23 & Epic 25 & Epic 27 & Epic Supra 19 & Epic Supra 21 & Epic Supra 23 \\
\hline \multicolumn{8}{|l|}{ LV mass, $\mathrm{g}$} \\
\hline \multicolumn{8}{|l|}{ MRI } \\
\hline Baseline & $128 \pm 19$ & $155 \pm 43$ & $170 \pm 35$ & $178 \pm 45$ & $163 \pm 72$ & $148 \pm 41$ & $150 \pm 46$ \\
\hline $6 \mathrm{mo}$ & $101 \pm 0.4$ & $114 \pm 28$ & $125 \pm 29$ & $131 \pm 25$ & $111 \pm 46$ & $111 \pm 26$ & $111 \pm 28$ \\
\hline \multicolumn{8}{|l|}{ TTE } \\
\hline Baseline & $207 \pm 48$ & $253 \pm 68$ & $264 \pm 63$ & $290 \pm 55$ & $240 \pm 68$ & $242 \pm 50$ & $246 \pm 46$ \\
\hline $6 \mathrm{mo}$ & $159 \pm 15$ & $168 \pm 38$ & $182 \pm 34$ & $207 \pm 32$ & $183 \pm 65$ & $180 \pm 38$ & $179 \pm 53$ \\
\hline \multicolumn{8}{|l|}{ LVMI, $\mathrm{g} / \mathrm{m}^{2}$} \\
\hline \multicolumn{8}{|l|}{ MRI } \\
\hline Basline & $72 \pm 10$ & $83 \pm 21$ & $89 \pm 18$ & $92 \pm 24$ & $97 \pm 46$ & $86 \pm 25$ & $82 \pm 20$ \\
\hline $6 \mathrm{mo}$ & $56 \pm 3$ & $63 \pm 13$ & $65 \pm 12$ & $67 \pm 14$ & $65 \pm 28$ & $64 \pm 16$ & $62 \pm 11$ \\
\hline \multicolumn{8}{|l|}{ TTE } \\
\hline Baseline & $121 \pm 31$ & $136 \pm 31$ & $139 \pm 34$ & $149 \pm 28$ & $141 \pm 41$ & $140 \pm 31$ & $137 \pm 20$ \\
\hline $6 \mathrm{mo}$ & $88 \pm 13$ & $94 \pm 15$ & $94 \pm 17$ & $106 \pm 18$ & $107 \pm 37$ & $105 \pm 26$ & $100 \pm 29$ \\
\hline
\end{tabular}

Values are means \pm standard deviation. For the different valve types and the valve sizes there is a significant regression of left ventricular $(L V)$ mass and left ventricular mass index ( $L V M I)$ comparing baseline to 6 months' follow-up $(P=.02$ for $19-\mathrm{mm}$ and $27-\mathrm{mm}$ valves, $P<.0001$ for all other valve sizes). MRI, Magnetic resonance imaging; TTE, transthoracic echocardiography.

TTE- and MRI-measured changes in LVMI between baseline and 6 months of follow-up.

\section{DISCUSSION}

AS causes LV pressure overload and a marked hypertrophic response of the myocardium as an adaptive response to the increased wall stress. ${ }^{6}$ This, in turn, causes an increase in LV mass, which is strongly correlated with an increased cardiac mortality and morbidity. ${ }^{4}$ Therefore, it is crucial in patients with severe, symptomatic AS to decrease the LV mass to normal levels. It has been previously observed that LV mass may not return to normal after AVR for AS because of other factors, such as poorly controlled hypertension or patient-prosthesis mismatch. ${ }^{6,7}$ In this study, an approximately $25 \%$ regression of LV mass and LVMI was observed with all sizes of both bioprosthetic models at 6 months after AVR, an observation concordant with the $15 \%$ to $38 \%$ decrease reported by previous studies. ${ }^{15-17}$ The impact of the various valve models and sizes implanted on the regression of LV mass remains controversial. Some investigators found a lesser decrease in LV mass with small than large valves, ${ }^{12,18}$ whereas others observed no correlation between valve size and regression of LV hypertrophy. ${ }^{19}$ In the meta-analysis by Kunadian and associates, ${ }^{20}$ the decrease in LV mass was similar among different valve types. In another study, Christakis and associates ${ }^{19}$ reported a similar regression of LV hypertrophy with 3 different valve sizes. In this study, a statistically significant decrease in LV mass and LVMI were observed with the 2 valve types and all valve sizes.

The primary objective of this study was to compare TTE with cardiac MRI in the assessment of decrease in LV mass and LVMI. Current clinical practices rely mostly on TTE for measurements of LV mass because it is readily available and correlates with contrast left ventriculography. ${ }^{11}$ However, although 2-dimensional echocardiography reliably measures LV wall thickness and LV diameter at the base of the heart, its calculation of the LV mass depends on the Devereux formula, which has limitations in diseased states because of the geometric assumptions it makes. ${ }^{9}$ Moreover, TTE is acoustic window-dependent and subject to considerable interobserver and intraobserver variability. ${ }^{13}$ In contrast, cardiac MRI yields precise and highly reproducible measurements. Several studies have confirmed the accuracy of cardiac MRI in the assessment of the hypertrophied left ventricle. $^{10,21-23}$ Bottini and colleagues ${ }^{21}$ found MRI to more precisely and reliably measure LV mass than TTE and to be more suitable to evaluate hypertensive patients. Allison and coworkers ${ }^{22}$ made similar observations in patients with hypertrophic cardiomyopathy, although in our study its seems that TTE grossly overestimated LV mass and LVMI, as observed in other studies. ${ }^{24}$

Another advantage of cardiac MRI is the volumetric quantification in the assessment of LV ejection fraction. TTE, which is based on geometric assumptions, detected a significant difference in LV ejection fraction between baseline and 6 months limited to the Epic Supra group. MRI recorded decreases in LV end-diastolic and endsystolic volume, thus no change in stroke volume, and statistically significant increases in LV ejection fraction between baseline and 6 months with both valve models, confirming its superiority versus echocardiography in the measurements of volumes. The decrease in follow-up cardiac output may be attributed to the slower heart rate, explained by the consumption of beta-adrenergic blockers in $50.3 \%$ of patients at 6 months versus $29.5 \%$ preoperatively.

Because of an approximately 10-fold higher cost of MRI compared with TTE, its routine use for estimating LV mass 
regression is still impracticable, especially as the absolute precision in its estimation is rarely needed. Despite this fact, MRI is a good alternative in patients in whom TTE is not possible or its quality is insufficient.

In conclusion, AVR using the Epic and Epic Supra bioprostheses results in a statistically significant decrease in mean LVMI at 6 months after the operation. On the basis of the results of this study and a review of the literature, it seems that MRI is more accurate in measuring the dimensions of the LV. An ultimate validation of this thesis could only be done comparing each of these modalities with pathologic examination.

We thank Dr F. Collart from the Cardiac Surgery Division of la Timone hospital, in Marseille, France, Professor R. Di Bartolomeo from the Cardiac Department of Policlinico Sant'Orsola Malpighi, in Bologna, Italy, Professor G. Laufer from the University Hospital, in Innsbruck, Austria, Mr Rodolphe Ruffy for the editing of the article, Ms Rita Ella Omega for the statistical work provided, and all our colleagues whose efforts have prominently contributed to this study.

\section{References}

1. Vahanian A, Baumgartner H, Bax J, Butchart E, Dion R, Filippatos G, et al. Guidelines on the management of valvular heart disease: Task Force on the Management of Valvular Heart Disease of the European Society of Cardiology; ESC Committee for Practice Guidelines. Eur Heart J. 2007;28:230-68.

2. Sedrakyan A, Hebert P, Vaccarino V, Paltiel AD, Elefteriades JA, Mattera J, et al. Quality of life after aortic valve replacement with tissue and mechanical implants. J Thorac Cardiovasc Surg. 2004;128:266-72.

3. Ali A, Patel A, Ali Z, Abu-Omar Y, Saeed A, Athanasiou T, et al. Enhanced left ventricular mass regression after aortic valve replacement in patients with aortic stenosis is associated with improved long-term survival. $J$ Thorac Cardiovasc Surg. 2011;142:285-91.

4. Levy D, Garrison RJ, Savage DD, Kannel WB, Castelli WP. Prognostic implications of echocardiographically determined left ventricular mass in the Framingham Heart Study. N Engl J Med. 1990;322:1561-6.

5. Ruel M, Al-Faleh H, Kulik A, Chan KL, Mesana TG, Burwash IG. Prosthesis-patient mismatch after aortic valve replacement predominantly affects patients with preexisting left ventricular dysfunction: effect on survival, freedom from heart failure, and left ventricular mass regression. J Thorac Cardiovasc Surg. 2006; 131:1036-44.

6. Villa E, Troise G, Cirillo M, Brunelli F, Tomba MD, Mhagna Z, et al. Factors affecting left ventricular remodeling after valve replacement for aortic stenosis. An overview. Cardiovasc Ultrasound. 2006;4:25

7. Imanaka K, Kohmoto O, Nishimura S, Yokote Y, Kyo S. Impact of postoperative blood pressure control on regression of left ventricular mass following valve replacement for aortic stenosis. Eur J Cardiothorac Surg. 2005;27:994-9.

8. Devereux RB, Reichek N. Echocardiographic determination of left ventricular mass in man. Anatomic validation of the method. Circulation. 1977;55:613-8.

9. Abramov D, Helmke S, Rumbarger Lel-K, King DL, Maurer MS. Overestimation of left ventricular mass and misclassification of ventricular geometry in heart failure patients by two-dimensional echocardiography in comparison with threedimensional echocardiography. Echocardiography. 2010;27:223-9.
10. Rajappan K, Bellenger NG, Melina G, Di Terlizzi M, Yacoub MH, Sheridan DJ, et al. Assessment of left ventricular mass regression after aortic valve replacement-cardiovascular magnetic resonance versus M-mode echocardiography. Eur J Cardiothorac Surg. 2003;24:59-65.

11. Myerson SG, Bellenger NG, Pennell DJ. Assessment of left ventricular mass by cardiovascular magnetic resonance. Hypertension. 2002;39:750-5.

12. Bech-Hanssen O, Caidahl K, Wall B, Myken P, Larsson S, Wallentin I. Influence of aortic valve replacement, prosthesis type, and size on functional outcome and ventricular mass in patients with aortic stenosis. J Thorac Cardiovasc Surg. 1999; 118:57-65.

13. Zoghbi WA, Chambers JB, Dumesnil JG, Foster E, Gottdiener JS, Grayburn PA, et al. Recommendations for evaluation of prosthetic valves with echocardiography and Doppler ultrasound: a report From the American Society of Echocardiography's Guidelines and Standards Committee and the Task Force on Prosthetic Valves, developed in conjunction with the American College of Cardiology Cardiovascular Imaging Committee, Cardiac Imaging Committee of the American Heart Association, the European Association of Echocardiography, a registered branch of the European Society of Cardiology, the Japanese Society of Echocardiography and the Canadian Society of Echocardiography, endorsed by the American College of Cardiology Foundation, American Heart Association, European Association of Echocardiography, a registered branch of the European Society of Cardiology, the Japanese Society of Echocardiography, and Canadian Society of Echocardiography. J Am Soc Echocardiogr. 2009;22:975-1014.

14. Masci PG, Ganame J, Francone M, Desmet W, Lorenzoni V, Iacucci I, et al. Relationship between location and size of myocardial infarction and their reciprocal influences on post-infarction left ventricular remodelling. Eur Heart J. 2011;32: 1640-8.

15. Brown ML, Schaff HV, Suri RM, Li Z, Sundt TM, Dearani JA, et al. Regression in left ventricular mass after aortic valve replacement for chronic aortic regurgitation is unrelated to prosthetic valve size. J Thorac Cardiovasc Surg. 2011;142:5-9.

16. Tomoeda H, Ueda T, Teshima H, Arinaga K, Tayama K, Fukunaga S, et al. Postoperative left ventricular mass regression after aortic valve replacement for aortic stenosis. Ann Thorac Surg. 2010;89:745-50.

17. Pibarot P, Dumesnil JG, Leblanc MH, Cartier P, Metras J. Changes in left ventricular mass and function after aortic valve replacement: a comparison between stentless and stented bioprosthetic valves. J Am Soc Echocardiogr. 1999;12: 981-7.

18. Hanayama N, Christakis GT, Mallidi HR, Rao V, Cohen G, Goldman BS, et al Determinants of incomplete left ventricular mass regression following aortic valve replacement for aortic stenosis. J Card Surg. 2005;20:307-13.

19. Christakis GT, Joyner CD, Morgan CD, Fremes SE, Buth KJ, Sever JY, et al. Left ventricular mass regression early after aortic valve replacement. Ann Thorac Surg. 1996;62:1084-9.

20. Kunadian B, Vijayalakshmi K, Thornley AR, de Belder MA, Hunter S, Kendall S, et al. Meta-analysis of valve hemodynamics and left ventricular mass regression for stentless versus stented aortic valves. Ann Thorac Surg. 2007;84:73-8.

21. Bottini PB, Carr AA, Prisant LM, Flickinger FW, Allison JD, Gottdiener JS Magnetic resonance imaging compared to echocardiography to assess left ventricular mass in the hypertensive patient. Am J Hypertens. 1995;8:221-8.

22. Allison JD, Flickinger FW, Wright JC, Falls DG 3rd, Prisant LM, VonDohlen TW, et al. Measurement of left ventricular mass in hypertrophic cardiomyopathy using MRI: comparison with echocardiography. Magn Reson Imaging. 1993;11:329-34.

23. Hunold P, Vogt FM, Heemann UW, Zimmermann U, Barkhausen J. Myocardial mass and volume measurement of hypertrophic left ventricles by MRI-study in dialysis patients examined before and after dialysis. J Cardiovasc Magn Reson. 2003;5:553-61.

24. Scharhag J, Urhausen A, Schneider G, Rochette V, Kramann B, Kindermann W Left ventricular mass in endurance-athletes with athlete's heart and untrained subjects - comparison between different echocardiographic methods and MRI Z Kardiol. 2003;92:309-18. 
TABLE E1. Incidence of clinical outcomes in 149 recipients of aortic Epic and Epic Supra bioprostheses at 30 days and 6 months of follow-up

Clinical events

Epic $(\mathbf{n}=77)$

Epic Supra $(\mathbf{n}=72)$

All patients $(n=149)$

0-30 days

Death

2 (2.6)

$2(2.6)$

$1(1.4)$

$3(2.0)$

Intrathoracic hemorrhage requiring reoperation

10 (13)

$2(2.8)$

$4(2.7)$

Atrial fibrillation

5 (6.5)

$2(2.8)$

$12(8.1)$

Complete heart block requiring permanent pacing

$3(3.9)$

$3(4.2)$

$8(5.4)$

Pericardial effusion

$0(0)$

$0(0)$

$3(2.0)$

Bleeding ulcer

$1(1.3)$

$1(1.4)$

$1(0.7)$

-6 month

Death

$0(0)$

$1(1.4)$

$2(1.4)$

Valve thrombosis

$0(0)$

$1(1.4)$

$1(0.7)$

Embolic stroke

$6(7.8)$

$1(1.4)$

$1(0.7)$

Withdrawal from the study

$8(10.4)$

$1(1.4)$

7 (4.7)

Miscellaneous causes of missing data

$60(77.9)$

$6(8.3)$

$14(9.4)$

Available data at 6 months

$61(84.7)$

$121(81.2)$

Values are numbers (\%) of observations.

TABLE E2. Comparison of patients who did versus did not undergo concomitant CABG surgery

\begin{tabular}{lcc}
\hline & \multicolumn{2}{c}{ Patient group } \\
\cline { 2 - 3 } \multicolumn{1}{c}{ Measurement } & $\begin{array}{c}\text { CABG } \\
(\mathbf{n}=\mathbf{2 6})\end{array}$ & $\begin{array}{c}\text { No CABG } \\
(\mathbf{n}=\mathbf{1 2 3})\end{array}$ \\
\hline Age, y & 73.3 & 73.3 \\
Body mass index, $\mathrm{kg} / \mathrm{m}^{2}$ & 28.4 & 27.8 \\
Body surface area, ${ }^{2}$ & 1.81 & 1.82 \\
LV echocardiographic & & \\
$\quad$ Ejection fraction at baseline, \% & 68.9 & 68.4 \\
$\quad$ Mass, g & & \\
$\quad$ At baseline & 240 & 253 \\
$\quad$ At follow-up & 178 & 180 \\
$\Delta$ LVMI, g/m & & \\
$\quad$ On echocardiogram & 35.8 & 38.8 \\
$\quad$ On MRI & 22.4 & 22.0 \\
\hline
\end{tabular}

Values are means. Between-groups differences are all statistically nonsignificant. $C A B G$, Coronary artery bypass graft; $L V$, left ventricular; $L V M I$, left ventricular mass index; $M R I$, magnetic resonance imaging. 